\title{
Prier, soulager : le chant des morts musical d'Encre plus rouge (2003)
}

Encre plus rouge est intimiste. Sans forcément se calmer, les eaux de l'inconscient - dont l'écume marque les cinq recueils précédents ${ }^{1}$ - nous bercent. Il est question des actes de parole que nous venons d'analyser, mais le souffle est plus ample, la progression d'idées plus souple et fluide, notamment grâce à la structure tripartite qui se tourne vers l'avenir par le biais de « la fable qui pourvoit » (ER 222), du songe comme champ de découverte continue. De nombreuses références au sacré et à la musique adoucissent le dire, renforçant l'impression qu'un « signe mat et clair » (188) viendra à notre secours, que la matière sonore et sémantique atteindra le cœur comme le ferait une « sonate » (218). On se pose avec Tellermann la question « Où demeurer [...] ?» (22), mais ce livre-poème est conçu, comme la trame d'une telle composition musicale, pour être un «sanctuair[e] » qui « délivr[e] / les feuilles du dedans » (24). Il laisse la place à l'association libre, pose des questions souvent sans réponse, fait se rencontrer de nombreux motifs et figures sonores, et suggère une éventuelle résolution des thèmes sans intégralement l'assurer.

Citant parfois Perse, adaptant par télescopage les rythmes surgissants et majestueux de son aîné à son propre regard sur le passé, le monde minéral, et l'ici et maintenant dans lequel s'enracinent les êtres lorsque le poème se fait chant, Tellermann approfondit sa méditation sur l'énigme qui nous habite. Elle avance par le dire, faisant retentir au fur et à mesure l'espérance. Exposer des thèmes en trois séquences comme elle le fait ici, au moyen de trames narratives qui s'entrecroisent sans être pleinement explicitées, en augmente les nuances et résonances. Laisser ouvert le sens accentue tant le sensoriel et le perceptuel que l'ouverture sur des circonstances réelles et mythiques. Son chant des morts

1 Voir par exemple la $4^{\text {e }}$ de couv. de $P A, T P, D F$ et $P G$. La « narratrice » de $P A$, bien que s'intéressant aussi au quotidien, médite « [l]'obscurité des racines » (69), laisse le sensible « apaise[r] un désir jusque-là inaccessible» ( $4^{\mathrm{e}}$ de couv.). Il est question dans $T P$ d'« une autre naissance », d'une oscillation « entre ascension et chute » qui rapporte «le personnage [...] à la grammaire de son identité »; dans $D F$, d'« itinéraires vers le "point central" qui se dérobe »; dans $P G$, de fragments s'inscrivant «sur la page intérieure où se profile [...] un seul texte invisible ». Aborder ces ouvrages par le biais de l'inconscient est une façon parmi d'autres - la fable, l'Histoire, les intertextes, le tissu allusif ou sonore, le tressage d'alliances - de saisir leur portée.

(C) AARON PREVOTS, 2022 | DOI:10.1163/9789004499683_004 
se fait tendre et sobre afin que nos gestes envers les disparus soient à la fois confiants et questionnants, chaleureux et irrésolus. La nomination indirecte et flottante, les failles et espacements dans les vers brefs, et l'enchaînement des séquences permettent au lecteur de rêver à son tour, d'admettre ce manque de clôture, ces « couleurs du dire / aux portes » (ER 223).

L'analyse suivante mettra en valeur la façon dont le devenir s'inscrit dans le dire. Tellermann aura beau traiter souvent des morts, son œuvre n'en reste pas moins vive et pleine d'entrain, ses recueils de plus en plus touffus et dialogiques. Divers éléments créent une musique certaine, aux niveaux des mots, du style et de la structure. Le tremblement du dire ${ }^{2}$ - les allusions, les hésitations, la polysémie, la reprise de motifs, l'aspect rituel des séquences et des gestes dépeints - infléchit celui-ci vers le sacré avec insistance. Dans Encre plus rouge, la beauté se trouve au premier plan, autant que le mystère ou les violences de l'Histoire. Appuyés sur un cadre narratif plus circonscrit et la fulgurance de l'encre rouge qu'est la matière du poème, le temps et l'espace peuvent alors se déployer. Nous nous plongeons dans le «pur présent $»^{3}$ d'une narration trouée, de personnages évoqués mais peu révélés, d'une terre sans nom dont se dévoilent les recoins.

Plusieurs spécificités, imbriquées les unes dans les autres, nous intéresseront: d'abord, les références implicites ou explicites à la poésie et au mythe, lesquelles mettent en relief tant la réflexivité de l'acte poétique que son urgence; ensuite, la singularité des images et de leur intrication dans le discours; enfin, la profonde intersubjectivité du dire tellermannien, où l'entrelacement d'images et de voix renforce l'idée que Tellermann prie pour ceux et celles à qui elle s'adresse, tâche de voir deux mondes. En considérant Encre plus rouge comme récit écrit pour « tisser littéralement [...] ce voile [...] dont on enveloppe les morts avant de les ensevelir ${ }^{4}$ nous reconnaîtrons des horizons spirituels et affectifs, y compris la pertinence des allusions à l'Antiquité et à la préhistoire. Nous saisirons comment, pour emprunter à Bernard Vargaftig (1934-2012) une devise que la lecture de Pierre Jean Jouve (1887-1976) lui insufflait, le mouvement du dire reprend celui de la musique, produisant

2 Cf. Aaron Prevots, « Pierre Jean Jouve et Bernard Vargaftig : tremblement et nudité du dire », Pierre Jean Jouve : dans l'atelier de l'écrivain, Dorothée Catoen-Cooche (sld), avec la collaboration de Béatrice Bonhomme, Myriam Watthee-Delmotte et Jean-Paul Louis-Lambert, Paris : Hermann, 2021, p. 257-74. On peut noter par exemple le soin porté par Pierre Jean Jouve, puis son disciple Vargaftig, aux qualités sonores et métriques des textes, ainsi que les variations autour de quelques thèmes de prédilection tels que l'éros, le dehors, le dedans et le sacré.

3 Anne Malaprade, « Entretien avec Esther Tellermann », op. cit., p. 165.

4 Ibid. 
des « espaces intérieurs » qui « engagent $[. .$.$] totalement l'être » : « Le sens se$ construit, pas à pas, $[\ldots]$ à travers une suite de variations toujours vivantes. ${ }^{5}$

\section{$1 \quad$ Réflexivité, poétologie, profondeur}

Les textes composés en regard ${ }^{6}$ par lesquels le livre s'ouvre annoncent que dire, ce sera faire. Leurs trois phrases morcelées anticipent sur «l'état d'urgence » dont il sera question dans la séquence initiale. D'une part, Tellermann émonde le dire pour mieux s'y engager: elle coupe les énoncés, en enlève l'inutile, débarrasse le vers de ce qui pèse, l'allège en incorporant des refrains. Elle précise qu'il s'agira d'une trajectoire rythmée par de nouveaux départs. Dès le mot clé « encore », semé dans l'injonction visuellement et syntaxiquement brisée qui commence le premier texte, elle indique l'immédiateté des propos qui vont suivre. Peut-être fait-elle le point après sa vingtaine d'années en tant que poète, se réjouissant de ce dont ce recueil traitera et laissant déjà entendre que des « feuille[s] d'or» (111) le composeront. Retenons surtout le côté physique des divers énoncés réflexifs : toucher « l'os », faire sentir « la cambrure » du monde et du dire, « aval[er] et crach[er] / le possible » de telle sorte que « notre soif » (8-9) soit étanchée. Réduire les mots à l'essentiel met en avant le travail que la langue poétique doit faire loin des discours courants, d'où la tonalité singulière de ces deux textes, au carrefour d'un sacré fougueux et indéniable d'un côté et, de l'autre, d'une modernité brisée, exténuée, chagrinée, soucieuse d'évoluer mais devant accepter son éternelle insatisfaction.

Par ailleurs, il y a un travail en filigrane pour faire fleurir le dire à travers des intertextes. Au fond, nous disent les poèmes d'Encre plus rouge, on n'est jamais seul. À travers le dire, l'Autre viendra à notre rencontre. Des voix vont s'entretisser afin de nous soulager et de nous redonner de l'élan. Tellermann parvient à les incorporer parce que ces voix viennent se greffer régulièrement sur le dire. Certes, le rouge renvoie à la passion qui nous habite tous. C'est la couleur du feu et du sang, symbole ambivalent exprimant autant l'énergie vitale et le mystère de la vie que le danger et la détresse. ${ }^{7}$ Employer tant cette couleur,

5 Bernard Vargaftig, «L'inflexion », Littérature suédoise, Europe 780 (avril 1994), p. 173-85 (181).

6 Isabelle Garron, «Esther Tellermann: Encre plus rouge», Action poétique 174 (2003), p. $74-75(74)$.

7 Voir Michel Cazenave, éd., Encyclopédie des symboles, Paris : Livre de poche, 2000, p. 592-94; Jean Chevalier et Alain Gheerbrant, éd., Dictionnaire des symboles : mythes, rêves, coutumes, gestes, formes, figures, couleurs, nombres, Paris : Robert Laffont, 1997, p. 831 ; Nanon Gardin et al., Petit Larousse des symboles, Paris : Larousse, 2019, p. 545-46. 
c'est se mouvoir dans ce monde symbolique, s'embarquer vers ces deux rives. ${ }^{8}$ On écoute toutes sortes de voix en naviguant avec l'auteur dans les «mondes éteints » (116) que de plus en plus elle affectionne, y compris celui, paradoxal, de la mort qui ne peut nous quitter, dont la présence nous rend humains. Mais ce recueil signale aussi un enjeu littéraire d'un autre ordre : rendre présentes d'autres voix en les incorporant de diverses manières à la matière du texte. Au-delà des réseaux sémantiques inhérents à la nomination, insister sur des allusions et reprises d'ordres divers met en branle un éventail d'émotions et de sensations. En soi, cette méthode n'est pas nouvelle. Tout un chacun, en écrivant, peut ou doit avoir à l'esprit des intertextes. L'important dans Encre plus rouge, c'est la façon dont Tellermann accède ainsi à la mort, fait perdurer en douceur les voix des disparus et met en évidence avec eux la musicalité et la sacralité des mots.

Poursuivons en reprenant le topos des intertextes, pour méditer la mémoire dans sa dimension littéraire, contempler l'inconscient dans sa dimension collective, en accueillir l'indicible. Indiquer quelques épissures nous montrera la richesse de la poétique de la différence - de l'exil, du fait d'être une étrangère ou un étranger, de la parole comme demeure, du deuil obviant au besoin de réparations - qui caractérise le dire tellermannien. En outre, observer ces épissures dans Encre plus rouge nous initiera au style mûr de Tellermann, à « l'intonation sinon réconciliée, du moins plus apaisée » $\left(4^{\mathrm{e}}\right.$ de couv. $)$ de sa voix. À ce propos, apparaissent de nouveaux enjeux critiques. Cela devient l'occasion de noter un foisonnement de sens et d'idées émanant de plusieurs ères, de voir quelques figures de l'étranger traverser ces lieux et espaces, d'entrer dans la fabrique de l'écrivain, là où coule l'encre comme le souffle. Nous mettrons l'accent sur un certain après-guerre littéraire, à savoir la prise de conscience chez Perse et Celan, dans la période 1940-1970, de ce que la poésie peut ou ne peut pas faire pour commémorer la vie et la mort et se distinguer comme discours.

Même la poésie la plus sombre, sévère ou consternée peut avoir des cadences et un élan, se jouer mezzo forte puis più forte ou mezzo piano, en augmentant ou diminuant son intensité. À l'instar de Perse, Tellermann allège le dire, adopte une palette colorée, tout en descendant plus bas tel Orphée parmi des

8 Une étude récente qui traite de la couleur dans la poésie française moderne et contemporaine (Mallarmé, Valéry, Bonnefoy) est Susan Harrow, Colourworks : Chromatic Innovation in Modern French Poetry and Art Writing, London : Bloomsbury, 2021. Harrow traite de liens qui se tissent à soi, à l'Autre, à la matière, au réel. En ce sens, son argumentation renvoie à l'emploi de la couleur chez Tellermann. Harrow montre que la couleur nous éveille au possible, au devoir éthique d'habiter poétiquement le monde, à la façon dont s'entremêlent l'affect et l'esthétique, le passé et le présent, la perception et la pensée critique, la sensation et le devenir humain. 
regrettés - gens, lieux, espaces, sensations, pensées - afin d'y voir et entendre une promesse. « Encore toucher / l'os » $(E R$ 8) : commençons par là pour examiner ce que le Perse d'Exil (1942) et de Vents (1946) apporte aux textures d'Encre plus rouge. Les textes de Perse apparaissent en filigrane en ce qui concerne une envie de chanter toute la terre et tous les êtres, d'envisager une vie passée auprès des morts et des vivants, avec vue sur tout versant et optimisme quant au pouvoir de la parole. ${ }^{9}$ Ces trois premiers mots d'Encre plus rouge, « Encore toucher / l'os », recèlent déjà des trésors qui seront révélés peu à peu. Au niveau lexical, ce vers fait écho à celui en prose - de quatorze syllabes si bien rythmés qu'il convient de penser en termes de vers - qui figure dans Vents, au début de la section II, 6 : «J'entends croître les os d'un nouvel âge de la terre. » ${ }^{10} \mathrm{Au}$ niveau sémantique, lire Tellermann en même temps que Perse fait paraître des objectifs similaires : chanter un nouvel âge, puiser dans le tellurique et en faire sa foi, recevoir comme un don les mots qui soufflent et croissent sur la page, toujours reprendre comme le feraient les vents réels et symboliques. Au niveau musical, Tellermann arrange autrement la partition qui en résulte. Savourons par exemple la retenue, la condensation, la «cambrure » (8) si différente de l'ossature des mots sur la page. Écrivant soixante ans plus tard, elle change de code, d'où l'idée de « crach[er] le possible» (9), adaptation peut-être du début de Cap au pire de Beckett : «Encore. Dire encore. [...] Encore et encore. Tant mal que pis encore. [...] Vomir et partir. ${ }^{11}$

Or, il ne s'agit pas d'un jeu vain. Chaque relique - l'os de tout mort, le mot en tant qu'os du dire - est sacrée, à garder près du cour, à conserver dans la mémoire. Depuis l'après-guerre, de nombreux poètes ont eu recours au mode citationnel, soit pour approfondir et contextualiser le dire comme ici, soit pour l'aplatir, le brouiller, ironiser. Dans Encre plus rouge, le dit parfois multiple amplifie l'urgence du récit. ${ }^{12}$ Les poètes du passé font partie intégrante des

9 Selon l'auteur, Perse l'a « autant accompagnée » que Celan à certains égards. Voir Aaron Prevots, « Entretien avec Esther Tellermann », op. cit., p. 110.

10 Saint-John Perse, CEuvres complètes, Paris : Pléiade, 1972, Vents, II, 6, p. 213. Toutes les citations de Perse viendront de ce volume.

11 Samuel Beckett, Cap au pire, Paris : Minuit, 1991, p. 7. Cf EC 27 : «Il voulait creuser [...] ce qui [...] vomit / un tréfonds. » Voir aussi Tellermann dans Anne Malaprade, «Entretien avec Esther Tellermann », op. cit., p. 163-64, à propos de la boue dont se charge la langue, ainsi que l'acte d'écrire et le rapport avec la langue comme réel que l'on crache et recrache. L'impossible - la béance, le trou, le vide - ne s'efface pas, mais l'œuvre tellermannienne va de l'avant, voit plus loin, continue toujours à creuser à l'instar de ses aînés. Cf. Michael Bishop, « Esther Tellermann : exactitude, interrogation, psaume », op. cit., p. 27-28, à propos de «l'insertion de l'humain dans l'énigme de l'ontos » (28). Vis-à-vis d'Anne-Marie Albiach, Catherine Soulier souligne le fait que «l'effet de citation [...] assure [...] la place de l'autre». Voir Catherine Soulier, «Résonances circulaires : notes sur L'EXCÈs : cette 
morts dont vient le chant. Cet aspect-là est souligné par d'autres strates intertextuelles. S'il faut encore «toucher / l'os » (ER 8), c'est que, selon Perse, il faut toujours tout « reprendre », « redire » la place et la fonction du poème comme «Livre », ajouter aux autres livres innombrables «par hautes couches crétacées portant créance et sédiment dans la montée du temps $» .^{13}$ Le poète doit aider le « grand arbre du langage peuplé d'oracles »14 à pousser. Dans Encre plus rouge, comme dans Vents, ce travail sera à faire « feuille à feuille » (ER 216), pour que surgisse des « écritures nouvelles » (ER 119). ${ }^{15}$ Chaque partie ajoutera à l'ensemble. Rien ne sera vide ou neutre. Si le rouge sera la couleur des nerfs, comme le suggère le titre de la deuxième section, c'est qu'il y aura une pulsation dedans, du sang et du souffle irriguant tout l'organisme. ${ }^{16}$ L'« incertain » (63), l'incertitude, l'inquiétude $(38,51)$, moteurs parfois du dire, seront un tremplin pour retrouver le possible, explorer soi et l'Autre, faire en sorte que l'aveuglement soit son complice. Une problématique clé sera de faire comme Perse malgré tout : quitter le domaine de l'histoire récente ; laisser de côté l'« attouchement » à son «doigt d'os » des «poudres du savoir » ${ }^{17}$; renouer souvent avec le versant ensoleillé de l'humanité, qu'il s'agisse de chaque instant auquel « naître » ou des « gîtes du futur » 18 ; et embrasser du regard « les terres rouges $[\ldots]$ la terre sigillée ${ }^{19}$ c'est-à-dire reconnaître la matière qui nous entoure, les

mesure (Anne-Marie Albiach - Richard Tuttle, 2004)», Anne-Marie Albiach, éd. Régis Lefort, $N U(e) 66$ (sept. 2016), p. 67-8o (74). Vents, I, 4, p. 186.

14 Vents, I, 1, p. 180. Au sujet du poème comme «Livre » chantant ceux et celles qui nous ont précédés, voir aussi $E R 228$ : «Berce-les / au grand Livre / / ou à l'envers. » Selon nous, « l'envers » voudrait dire par exemple le silence plein qui peut accompagner la parole, ou bien la vraie vie, en dehors de l'écrit.

Cf. Vents, II, 6, p. 214, où de « grandes œuvres » qui se composent « feuille à feuille » nous donnent des « écritures nouvelles».

« [L]e sang joue un rôle nutritif, respiratoire et régulateur essentiel. [...] [V]éhiculant des valeurs de noblesse, de générosité, d'hérö̈sme », il est « constitutif de l'existence même de l'être humain » (Nanon Gardin et al., Petit Larousse des symboles, op. cit., p. 557).

Vents, I, 4, p. 187. Cf. Vents, III, 4, p. 224 : quitter le domaine du savoir afin de parler de «présence[s] humaine[s]», de «hautes mers intérieures», de l'homme pour qui «le Poète » peut «témoign[er] », en reconnaissant ce que nous apporte le vécu profond de l'humanité - les « chambres millénaires».

18 À propos de l'instant auquel naître, voir Vents, I, 2, p. 181; Vents, III, 6, p. 229 ; et Vents, IV, 5, p. 248 (cf. ER 128). Perse traite des « gîtes du futur» dans Vents, II, 6, p. 214 (cf. les «vasques du futur», Vents, I, 2, p. 181). Le poète parle pour l'humanité « [c]ar c'est de l'homme qu'il s'agit, et de son renouement » (Vents, III, 4, p. 226).

19 «Et les terres rouges prophétisent sur la coutellerie du pauvre. Et les textes sont donnés sur la terre sigillée » (Vents, II, 6, p. 214). Sigillé, e : «(lat. sigillum, sceau). Marqué d'un sceau ou d'une empreinte semblable à celle d'un sceau. (Se dit d'une céramique gallo-romaine rouge, vernie et ornée d'un décor en relief)» (Georges Lucas et al., éd., 
cycles du temps au sein desquels nous vivons et le poème comme fusion avec l'élémentaire.

Arrêtons-nous un moment sur cette tonalité rouge. N'oublions pas l'alternance systole-diastole du cœur humain. Si le Perse d'Exils et de Vents irrigue les textes d'Encre plus rouge, il y aura néanmoins de nombreuses variations sur ses thèmes. En relisant Perse, nous apprenons que l'ambiguïté du dire Tellermann s'insère dans le cadre d'une poétique de l'équivoque. ${ }^{20}$ Perse sert d'exemple pour établir ce cadre, mais Tellermann marche à son propre rythme, d'un pas plus lent et modeste. Elle se met à l'écoute de son aîné afin d'invoquer les morts (diastole), mais façonne en même temps ses propres mythes (systole), hésitant, bien entendu, à être ce «Shaman sous ses bracelets de fer: / Vêtu pour l'aspersion du sang nouveau $»{ }^{21}$ Elle se plonge dans le verset persien, mais, au moment d'écrire, penche pour la litote, l'espacement, la cristallisation, tout en privilégiant comme lui le caractère ouvert, irrésolu, insituable du dit. On peut constater ainsi l'« autonomie de la création $»^{22}$ que revendique Perse, reprise sous d'autre formes, la solitude et « l'exploration nomade et vaine $»^{23} \mathrm{en}$ moins, l'amour et la conscience exprimés plus souvent sur le ton de l'intimité. ${ }^{24}$

En effet, Perse l'accompagne presque physiquement, en tant que confrère au même office. Parfois, c'est lui, l'Autre, prenant la parole à tour de rôle avec Tellermann. Prendre en compte ces intertextes, c'est voir le Poète - la majuscule vient de lui - comme âme sœur, le dire littéraire comme « orage » qui nous « lav $[\mathrm{e}] »(E R 35) \cdot{ }^{25}$ Se faire accompagner par lui, c'est se faire guider pour reconnaître les liens profonds entre l'énigme et l'être, la prière - même la plus humble - et ce que nous serons. Ensemble, ils « fond[ent] sur l'abîme et l'embrun et la fumée des sables », «épie[nt] au cirque le plus vaste l'élancement

Petit Larousse illustré 1984, Paris : Larousse, 1984, p. 931). Notons aussi qu'un texte 'sigillé' pourrait s'associer au sacré, aux échanges profonds avec la terre ou avec des personnages mythiques.

Vents, II, 6, p. 213 : « homme parlant dans l'équivoque !... ah ! comme un homme fourvoyé dans une mêlée d'ailes et de ronces, parmi des noces de busaigles !».

21 Vents, I, 2, p. 181 ; cf. ER 35 .

22 Colette Camelin, «Les CEuvres complètes de Saint-John Perse », Modernité de Saint-John Perse? Actes du colloque de Besançon des 14, 15 et 16 mai 1998, éd. Catherine Mayaux, Besançon : PU Franc-Comtoises, 2001, p. 251-64 (256). Jacques Darras, «L'énigme et la déception. Poèmes présentés par Jacques Darras », $<$ esprit.presse.fr>.

24 Charles Dobzynski, «La vie au plus près : Andrée Chedid, Esther Tellermann », Robert Walser, Europe 889 (2003), p. 334-38 (337).

25 Cf. Vents, I, 2, p. 181 : « Ô vous que rafraîchit l'orage... Fraîcheur et gage de fraîcheur... »; Vents, IV, 6, p. 250 : « Ô vous que rafraîchit l'orage, la force vive et l'idée neuve rafraîchiront votre couche de vivants ». 
des signes les plus fastes $»,{ }^{26}$ par exemple là où Tellermann insiste de manière ambiguë sur la couleur rouge, reprend des figures sonores comme ces allitérations en [f], cite un syntagme inattendu tel le "goût d'airelles / pour naître » $\left(E R{ }_{128}\right),{ }^{27}$ ou emploie les pronoms tu, nous, il et ils sans préciser à qui ces pronoms se réfèrent. Sans oublier l'éclat et le hiératisme du dire persien, son élégance rhétorique, il faut remarquer comment cette épaisseur du dit ouvre une porte sur les abîmes et embruns de l'âme et du langage, pour que l'on parle mieux « aux vivants ${ }^{28}$ d'autrui, des êtres et des choses, de ce qui se passe sous terre ainsi que dans l'imbrication des saisons, des vents, des générations.

D'une part, l'« organisation précise » chez Perse mène à de « secrets rapports » : lui faire allusion, et à travers lui penser par exemple à un Hugo ou à un Nerval, « aux forces obscures de la Nuit », ${ }^{29}$ est pour Tellermann une façon de mieux pénétrer dans le réel, de peupler le récit, de créer de vastes étendues spatiotemporelles. De l'autre, il est difficile de ne pas constater dans Encre plus rouge un côté ludique, un imaginaire plus développé que celui de Guerre extrême et qui ose reprendre des topoï persiens en adaptant à ses fins leur tonalité. L'« envers du livre » (ER 9) peut être les « fruits ouverts » (15) que sont les mots. Lorsque les «frères se renvers[ent] » (20; cf. 21, 23), il semble être question de compagnons de route, d'autres auteurs qui ont souffert. Une allusion au côté cérémonial du dire persien, «Brûlions-nous les signes / nos façons de tendre / bracelets jambes / taches de l'infini ? » (35), semble teinté de regret ou de nostalgie. Bref, accompagner d'autres auteurs, c'est aussi changer le ton ou le mode de leur dire, le mettre à jour. De plus, lorsque Tellermann se met en dialogue avec eux, l'ouverture de ses allusions - leur ambivalence sur le plan symbolique lorsqu'elles s'insèrent dans cette nouvelle étoffe textuelle crée des hésitations musicales en introduisant des échos, de nouveaux horizons sonores, voire d'autres harmonies. Ainsi l'« encre plus rouge » devient-il un élément clé de l'oscillation entre des pôles affectifs. Le rouge de ce livre s'accorde au battement du cœur qui a lieu lorsqu'on pense aux morts et souhaite les admirer autant que les pleurer, souligner ce qu'ils continuent à nous

$26 \quad$ Exil, « Exil », p. 124 et p. 125 .

27 Vents, III, 6, p. 229 : «Quel est ce goût d'airelle, sur ma lèvre d'étranger, qui m'est chose nouvelle et m'est chose étrangère ?... ».

28 Vents, I, 2, p. 181.

29 Colette Camelin, «Les CEuvres complètes de Saint-John Perse », op. cit., p. 26o. Vents, II, 6, p. 214 : «Et toi, Soleil d'en bas, férocité de l'Être sans paupière, tiens ton œil de puma dans tout ce pain de pierreries !... »; Vents, III, 5, p. 228 : « Contribution aussi de l'autre rive ! Et révérence au Soleil noir d'en bas ! ». ER : «Soleil noir / ferme les portes de Jérusalem » (141); « J'ai vu / des soleils noirs / des rivages hirsutes » (180) ; « soleil noir s'est levé / aux portes de Jérusalem » (200). 
apporter autant que le fait qu'ils nous manquent. Faire des emprunts à Perse et lui faire allusion sont des procédés qui servent, comme celui de parler souvent des « couleurs du dire » (223), à mieux interroger soi et le langage quant au désir d'atteindre l'Autre.

Si la terre est « bigarrée comme un cri », c'est justement parce que Tellermann veut instaurer «un accord» qui permette aux morts de «se balanc[er]», d'ajouter leurs voix à la prière qui rend à l'humus sa « fraîcheur » (73). La voix de Celan est plus sombre, son urgence mêlée souvent de vide à cause de l'Histoire à laquelle elle doit faire face. La diction se réduit à l'essentiel, selon une logique de la réappropriation d'une langue porteuse de violence et de ténèbres, un processus de reformulation ou de recomposition de celle-ci. ${ }^{30}$ Le poème construit des réseaux de sens en privilégiant l'ellipse et la polysémie, en multipliant les points de vue à partir de mots méticuleusement choisis, réfractaires, irréductibles. ${ }^{31}$ Le poète, un écorché vif contraint à vivre l'exil à même la langue dans laquelle il écrit, se tient à distance du lecteur, dans un autre espace-temps, accentuant la densité des mots et évitant de se présenter comme voyant ou prophète. ${ }^{32}$ Il s'agit de persévérer malgré l'exil et la différence imposés du dehors, de témoigner encore pour soi, ses proches, un peuple, la langue dont les semailles sont incertaines. ${ }^{33}$ Le rouge celanien s'offre à nous, à travers une Parole quelquefois à la limite du bégaiement, à la fois comme fleur tendue aux

30 John E. Jackson, Paul Celan : contre-parole et absolu poétique, Paris : Corti, 2014, p. 12, p. 19 et p. 29. Cf. Paul Celan, « Révolte de banderoles de brume, de banderoles de sentence », Renverse du souffle, trad. et éd. Jean-Pierre Lefebvre, Paris : Seuil, 2006, p. 177, où il est question de marteler le langage afin que le rouge et l'or soient luisants.

Frédéric Marteau, «Lire - ne pas interpréter ? Paul Celan et la résistance du texte poétique », La Posture de l'herméneute : essais sur l'interprétation dans la littérature, éd. Alison Boulanger et Jessica Wilker, Paris : Classiques Garnier, 2011, p. 27-43 (37) : « Celan [...] rappelle l'exigence critique du poème et la distance nécessaire (et congénitale) qui le sépare de son lecteur(-prédateur), où il s'agit d'assumer la singularité d'une parole, son étrangeté, son "lointain", son obscurité : cette irréductibilité de la parole poétique, il la conçoit à travers une grille, une grille de parole (Sprachgitter) qui empêche tout risque d'appropriation. »

Cf. Hugo Huppert, «"Spirituell”. Ein Gespräch mit Paul Celan », Paul Celan, hrsg. von Werner Hamacher und Winfried Menninghaus, Frankfurt am Main : Suhrkamp, 1988, p. 319-24 (321), entretien où Celan explique cette distanciation par rapport au lecteur, cette accentuation de la densité des mots, ainsi que l'exégèse de cet entretien dans Frédéric Marteau, «Lire - ne pas interpréter ? Paul Celan et la résistance du texte poétique », op. cit., p. 37-39, dont nous venons de citer un extrait.

33 Cf. Paul Celan, « Argumentum e silentio » [Pour René Char], De seuil en seuil, trad. Valérie Briet, Paris : Christian Bourgois, 1991, p. 110-13. 
disparus, trace de «ce qui fut silencié » et «sang » 34 inscrit dès lors dans le dire. Vis-à-vis de l'analyse de Perse ci-dessus, cette couleur correspond aussi pour Celan, comme nous le rappelle Martine Broda, à «l'Homme, qui survit au-delà de la mort des hommes, comme l'espoir survit à la mort des matins » ; à «la rose lyrique» de la «sensibilité élégiaque allemande» qu'il faut railler; et à une « rose des vents » qui peut consoler en volant librement comme don fait infiniment au lecteur malgré «la lumière noire de la déroute.$^{35} \mathrm{La}$ « cambrure » $(E R 8,140)$ dont parle Tellermann devient donc non seulement ondulation tellurique et élémentaire, mais aussi place faite aux morts courbés sous le poids de la souffrance, à l'Homme voulant garder tout de même en mémoire la nuit dont « retentissent » les chaînes. ${ }^{36}$ Autant de réverbérations, en somme, dans l'espace textuel d'Encre plus rouge dès les premiers vers.

Par ailleurs, ces nombreuses strates intertextuelles ont des dimensions de plus en plus musicales et poétologiques. Elles permettent au sujet lyrique de parler sans devoir «faire sens», de contourner tout «sens précis ».37 L'envie de faire des juxtapositions devient encore plus évidente. En creux, par le biais d'allusions, diverses cadences et tonalités coïncident. La fierté et la grandeur voisinent avec l'introspection et le questionnement. De fins discours sur soi doivent respirer le même air que ceux, plutôt angoissés, qui sont partagés à voix basse. L'auteur joue ainsi diminuendo et rallentando, en diminuant le son et en ralentissant, nous obligeant d'apercevoir ces contrastes, d'y réfléchir, de prêter notre attention aux morts en profondeur, avec concentration, voire reconnaissance et délices. Cela semble être le sens des vers «je briserai / la note pleine / dans ma cambrure » : accepter « les barques lentes », que l'auteur soit une sorte de « Cassandre » ou « Délie pieds nus » (140), emblème du désir

34 Ibid., p. 110 (nous traduisons). Cf. «Une étoile », Enclos du temps / Zeitgehöft, op. cit., p. 53. Quant au bégaiement, voir notre discussion d'EC dans le chapitre 6, vis-à-vis du poème de Paul Celan «Die Silbe Schmerz » / « Les syllabes douleur», La Rose de Personne, op. cit., p. 130-33. Cf. « Tübingen, janvier», La Rose de Personne, op. cit., p. 39, et Andréa Lauterwein, «Paul Celan : le rossignol bègue. Un changement de perspectives dans la poésie », Revue d'Histoire de la Shoah 201 (2014/2), p. 403-30, <cairn.info >.

Dans Paul Celan, La Rose de Personne, op. cit., p. 179, p. 181 et p. 184-85. Quant à la « rose des vents » (Windrose), voir « Die Silbe Schmerz / Les syllabes douleur», p. 130-33. Voir aussi $E R$ : «l'hibiscus » (160), « le couchant pourpre » (177), « Il y eut le sang / il y eut l'os » $(187,191)$ et « la rose » $(209)$.

36 Vis-à-vis de gens souffrants, courbés, dont le cœur a pu être percé, voir Paul Celan, «(Je te connais », Renverse du souffle, op. cit., p. 47. Quant à la nuit dont « retentissent » les chaînes, cf. «Argumentum e silentio » [Pour René Char], De seuil en seuil, op. cit., p. 110 (nous traduisons). Jacques Darras, « L'énigme et la déception », op. cit. 
mais non de promesses toujours tenues. Il faudrait plutôt diriger le regard vers «l'autre profondeur » (140), implicitement celle des deux versants du réel toujours présents, des sens pleins mais provisoires, par exemple l'appréhension que les morts et les vivants s'accordent et ont besoin les uns des autres, ce qui leur donne tous une « cambrure », de la fierté, une belle allure. De même, le savoir des poèmes d'Encre plus rouge est poétologique, intersubjectif, ancré dans le dépliement de rapports. C'est du poème comme lien vers l'Autre qu'il s'agit, des mots assez aimantés pour créer du relationnel. Permettons-nous une dernière remarque sur les deux textes liminaires composés en regard, pour souligner par le biais de la psychanalyse comment la prière du poème nous soulage, non sans un certain érotisme: «Encore, c'est le nom propre de cette faille d'où dans l'Autre part la demande d'amour. ${ }^{38}$ Le rouge éponyme, c'est souvent la couleur de ce mouvement, de lénigme du désir - également sous la forme de l'amour ressenti pour ceux et celles qui ne sont plus parmi nous surgissant de l'inconscient. ${ }^{39}$

\section{Séries d'images et polysémie}

Quelles sortes d'espaces intérieurs peut-on constater dans les trois séquences? Quelles images sont aussi polysémiques que cette « encre plus rouge » ? Comment est-ce que Tellermann engage totalement l'être à travers l'étoffe textuelle? Cette partie de notre analyse abordera une autre spécificité, celle des séries d'images qui relient de nombreux vers, qui retentissent sur lâme du lecteur de par leur regroupement, leurs sens multiples et leur structure souvent triadique. Si l'un des objectifs du recueil est de tendre la main aux morts, ce trait saillant y ajoute toutes sortes de textures, de profondeurs, de couleurs, d'harmonies. Il ne sera pas possible de les considérer toutes. Bornons-nous à étudier l'agencement des images et la manière dont le récit se développe dans la première section. Chemin faisant, nous découvrirons combien les séries d'images mettent en avant une prière pour l'Autre et servent à réconforter énigmatiquement le lecteur. Nous verrons que la signifiance l'emporte sur le

38 Jaques Lacan, cité dans Isabelle Garron, «Esther Tellermann : Encre plus rouge », op. cit., p. 74. Cf. Jacques Lacan, Encore [Le Séminaire, Livre XX, 1972-1973], éd. Jacques-Alain Miller, Paris : Seuil, 2016. Pensons également au mot d'André du Bouchet « tout est dit / mais il faut le répéter sans cesse, comme on respire » (Carnet, Saint Clément: Fata Morgana, 1994, p. 11 [avril 1951] ; cf. Une lampe dans la lumière aride, éd. Clément Layet, Paris : Bruit du temps, 2011, p. 78-79).

Cf. Nanon Gardin et al., Petit Larousse des symboles, op. cit., p. 545 : « Feu et sang, le rouge est la couleur du mouvement. » 
sens, puisqu'elle fait durer diverses relations : avec le temps et l'espace, la vie et la mort, le langage et autrui.

Nous venons d'examiner le sens comme mouvement grâce aux tonalités qu'apportent les intertextes. Poursuivons la lecture en remarquant comment le sens se construit pas à pas au moyen de l'image. Seul bémol : il nous faudra au passage continuer à parler de temps à autre d'allusions. Sur le plan de l'image, les allusions font vibrer des harmoniques, des sons accessoires qui se surajoutent au son fondamental, le tout donnant le timbre. Qui a entendu le chant dans une église gothique, dont les grands espaces font résonner de façon apaisante les voix, comprendra bien cette perception de fréquences complémentaires. Il importe de signaler cet aspect-là à cause du dispositif réduit ${ }^{40}$ d'où jaillit l'image. Les vers sont courts et troués. Peu de choses sont expliquées. Geste et parole font un ${ }^{41}$ Le rite et ses formules sertissent le dit, rendent caduque toute précision. L'objectif est à la fois de façonner un lieu qui ouvre sur l'histoire et de laisser à l'insaisissable sa part. Tellermann réduit donc le cadre et le parsème de « mots-cailloux ${ }^{42}$ ressemblant aux fleurs celaniennes dont nous venons de parler, à l'embrun persien qu'emportent les vents. Il peut être difficile de percevoir la valeur des sens conjointement possibles, le rôle du propre et celui du figuré. La superposition polysémique ${ }^{43}$ peut facilement dépasser deux sens, voire favoriser le figuré, s'appuyer sur l'emploi abstrait d'un vocable chez d'autres auteurs. Dans les observations suivantes, figureront parfois dans les formules analysées de telles harmoniques, autant de luminosités qui embrasent le sens et suscitent la réflexion.

Le premier texte de la section «État d'urgence » évoque la venue en présence de la parole : «Quand survinrent / nos désirs de sucre / ciel s'offrit / en fruits ouverts » (ER 15). Les allitérations et les «mots simples du musée universel de la poésie $»^{44}$ nous mettent sur terra firma par rapport aux énoncés plus abstraits des textes initiaux. La parole, en tant que mots venant de l'inconscient et de l'élémentaire, a été donnée abondamment à un Nous, « en fruits ouverts » à savourer, en mots qui comptent et racontent nos envies, peut-être un « chargement de fruits nouveaux $»^{45}$ comme ce serait le cas chez Perse. Et pourtant,

40 Charles Dobzynski, «La vie au plus près : Andrée Chedid, Esther Tellermann », op. cit., p. 337 .

41 Ibid. Cf. Michel Apel-Muller, « "Je ne crois pas à l'éternité de la parole" : Michel Apel-Muller s'entretient avec Bernard Vargaftig », France Nouvelle 1576 (26 janv. 1976), p. 28-30 (29).

42 Ibid., p. 337.

43 Michèle Aquien et Georges Molinié, Dictionnaire de rhétorique et de poétique, Paris : LGF, 1999, p. 629 .

44 Jean-Pierre Lefebvre, dans Paul Celan, Choix de poèmes, op. cit., p. 19.

45 Vents, IV, 6, p. 251 : « Un très vieil arbre, à sec de feuilles, reprit le fil de ses maximes... [...] Avec sa feuille magnétique et son chargement de fruits nouveaux ». 
nous sommes peut-être sur terra incognita. Avant, une série d'impératifs créait de l'immédiateté ; ici, le passé simple nous ralentit, nous éloigne quelque peu de la notion si dynamique de « $\operatorname{crach}[\mathrm{er}]$ le possible » (9). La phrase suivante, « Ferveur dut nous jeter », laisse planer le doute, suggère un renversement ou une déception. Le ton noble connote une douleur qu'il sied d'accepter. Les derniers vers, «Qui réduirait / l'urgence / et tous nos soirs assis ? », peuvent nous confondre à cause de leur ambiguïté. La phrase est à la fois une question et un appel, présentée en code énigmatique sans recours aux mots simples et sans rimes. Qui fut assis, et pourquoi ? Les «soirs assis» sont-ils synonymes de paresse ou de planification, de calme ou d'orage? «[L]'urgence » est-elle l'envers des invocations précédentes, l'aveu d'un mal-vivre, de ce qui manque et qui va être exploré ? Est-il question de maîtriser sa ferveur, ou «l'urgence » est-elle, au contraire, valorisante et nécessaire, une condition préalable pour recevoir en don les « fruits ouverts » de la parole?

Finalement, Tellermann nous invite tout simplement à rester à l'écoute du dire pour mieux devenir. Pour le lecteur, il s'agit de suivre ce dépliement de la pensée et de la parole. Il faut certes relier les diverses images, mais cela se fera au fur et à mesure, d'une page à l'autre et de séquence en séquence. La barque poétique partira loin. Le « soulas » (76) viendra si l'on reste attentif aux hauts et bas de l'histoire. «Chante[r] plus haut », c'est « supposer » des « frontières » et des « seuils » à traverser (139), les rebondissements de ce récit fragmentaire. Le rythme et les gestes de la trajectoire, l'interrogation de soi, de l'Autre et du langage, seront aussi importants que le sens. Tout ce qui relève de l'oracle se résoudra provisoirement avec le temps. Dans ce cas-ci, il faut noter les cadences et les couleurs sonores du dit, puis regarder en arrière et en avant pour saisir les énoncés. Les allitérations en [s], par exemple, relient entre elles les images du début et de la fin de «Quand survinrent » : les évocations d'un ciel qui s'offre à nous et de désirs qui soient « de sucre » font ainsi partie de «l'urgence » qui sera le fil conducteur. Le rythme ternaire des énoncés « fruits ouverts » et « soirs assis » nous rend attentifs au déploiement du temps et de l'espace, valorisant l'en-avant de la parole. Il en va de même pour les derniers énoncés, de six syllabes chacun : « Ferveur dut nous jeter »; « Qui réduirait / l'urgence »; « et tous nos soirs assis ». Si l'on regarde assez loin en arrière, cela fait penser à Distance de fuite: «- Je n'avais pour prier que le balancement. / / / Elle a reporté sa douleur » ( $D F$ 68). Si l'on considère le texte initial, « ce que nous voulons / et crachons / et notre soif » $(E R 8)$, on constate que le désir nous fait vivre, sert de repère. Regarder en même temps les pages suivantes nous aide à accueillir les séries d'images, ainsi que leurs rythmes respectifs en termes de syllabes et de nombre d'images. Cela montre combien il s'agit de structures constitutives, d'une ouverture aux cadences du dire et de la pensée. On commence à glaner 
des sens supplémentaires, par exemple le poème comme terre mentale où se rapprochent des réalités plus ou moins éloignées, puis l'« urgence » d'accepter le mouvement de «bascule » (17) que cela entraîne. En somme, suivre le flux et le reflux des images, comprendre leur agencement ainsi que le souffle qui nous les apporte, nous rappelle que le sens est toujours à venir, inscrit dans le déploiement du dire pour que celui du temps et de l'espace ait lieu aussi.

Les rythmes de la pensée se révèlent vite centraux. Ce principe organisateur comprend l'affect et les allusions, le songe et le mythe, la douleur et la joie. La compacité et la syntaxe souvent suspendue ${ }^{46}$ ajoutent à la charge émotive, comme le font les nombreuses questions posées et de brefs dialogues désignés par des tirets. Si la violence et la mort font surface, c'est dans le contexte de cet intimisme, de remarques presque sotto voce, par exemple à propos du «tragique / de 2 versants » (77) auquel acquiescer, d'une marche à la recherche des morts (114), de ceux et celles qui sont, au-dedans de nous, « fondus à perte / / éployés dans l'Amen » $(127 ; \mathrm{cf} .98,123)$. Le silence, le secret et le non-dit ne sont pas loin du dit: « tout mot t'arrache / un peu d'orage / chaque mot écrit / est un mot prononcé / soustrait » (91). C'est ainsi que les rythmes et les couches de sens des mots fascinent le lecteur, nous font quitter le silence tout en laissant bon nombre d'idées implicites, en suspens, à retrouver comme autant de fils d'Ariane. Il y a même des commentaires métatextuels qui se réfèrent à ce phénomène : « linges battent les temples / les 4 types de désert » $(52) ;$ « nous avons bercé les princes / aux 4 dimensions » (199); « Qu'est-ce qu'une pensée / parvenue ?» (211); « nous irons / plus haut que nous / léguant / / les 4 portes » (225).

Dans ce dire hiératique et réflexif, la polysémie est omniprésente mais organisée, rythmée, orientée vers la découverte de soi, de l'Autre et du possible que recèle et dévoile le langage. Souvent c'est comme si Tellermann dressait un bilan poétique, un état des lieux prenant en compte quelques-uns des écrivains qu'elle admire et leur élan contre des écueils ou des tribulations. Nous en avons parlé quant aux « frères » qui «se renvers[ent] » (20). Regardons aussi du côté des séries de deux ou trois images dans la section «État d'urgence » (11-57), en ce qui concerne l'épaisseur que créent les images et l'hommage à ceux qui « cuisent / les écritures / et sont cousus » (54), c'est-à-dire qui restent attentifs aux signes, les refondent dans le poème, se guérissent et se recréent à partir de fils poétiques. Qu'elles soient terre-à-terre ou abstraites, ces séries

46 Jean-Marie Perret, «Esther Tellermann, Encre plus rouge», op. cit., p. 114; cf. Michael Bishop, « Esther Tellermann. Encre plus rouge », World Literature Today 78.3 (May-Aug. 2004), p. 85-86. 
s'avèrent hypnotiques sur les plans affectif, rythmique, sémantique, sonore et visuel. L'œil et l'esprit en particulier sont attirés par ce qui se dérobe.

Les tropes celaniens peuvent nous confondre au premier abord, mais s'éclairent à la réflexion. Perse, dont la parole est moins cryptée, semble rôder alentour. Chaque énoncé exige une grande acuité de pensée. Prenons en exemple ceux du deuxième texte, qui commence par « Linge qu'il déceint / pour soutenir / le vide » (16). Le lexique ne surprend pas tellement. Le registre en est soutenu, mais compréhensible. Lérotisme se laisse facilement deviner, quoique « linge » se distingue de «vide », ce mot-ci appartenant plus au lexique celanien ${ }^{47}$ celui-là étant plutôt persien. Ensuite, la phrase « Au lieu serré l'étrange / nous renversa » peut exiger des explications. L'image « [un] lieu serré » n'emprunte pas particulièrement à un domaine reconnaissable : peut-être l'érotisme, le souffle, «l'urgence » (15) dont il est question et donc un sentiment de pression, les « soirs assis » (15) qui rendent le lieu du poème habité, peuplé, « serré » (16). Or, le deuxième énoncé « l'étrange nous renversa » vient à notre secours, met en regard les deux syntagmes aux niveaux sonore - [l], [s], [R] / [l], [R], [s] - et poétique. Dans l'univers celanien, les fils du poème, très serrés, s'opposent résolument aux discours contemporains. La matière poétique doit même avoir assez d'étrangeté pour nous renverser : nous faire voir, penser, souffler autrement, nous éloigner même de la métaphore et de l'image. Enfin, les vers 6 à 12 vont exactement dans ce sens-là : il y a renversement jusquà la « natte » qu'est le poème lorsque ses fils s'entrelacent comme il faut; jusqu'à la «jonction / de la natte et du deuil », en ce qu'il faut patiemment en arriver à parler aux morts et de la mort ; jusqu'aux «pluies » et à « l'irruption » lorsqu'il devient possible de partager correctement dans ce cadre ce que l'on ressent. ${ }^{48}$

47 Chez Celan, un centre charnel ou métaphysique peut être à la fois « vide » et « hospitalier » («À main droite et main gauche», La Rose de Personne, op. cit., p. 25); des « formes vides » peuvent faire penser aux disparus et à leurs voix sans mots ( « Les syllabes douleur», ibid., p. 131); et des cruches peuvent porter à la bouche «le vide [...] comme le plein» («Les cruches», [Pavot et Mémoire], Choix de poèmes, op. cit., p. 69). Autre exemple : «Strette», Grille de parole, op. cit., p. 91-105 (95-99), où il s'agit d'une parole à peine perceptible, « cendre », « Nuit-et-nuit », d'« un reste à chercher ». Quant à Perse, voir par exemple Exil, « Neiges», p. 163 et Vents, IV, 6, p. 250 : «Quelle navette d'os aux mains des femmes de grand âge, quelle amande d'ivoire aux mains des femmes de jeune âge / / nous tissera linge plus frais pour la brûlure des vivants ? » ; « Ô vous que rafraîchit l'orage, [...] l'odeur fétide du malheur n'infectera plus le linge de vos femmes ».

48 À propos de la suppression de la part de Celan de tout ce qui facilite ou accélère la lecture du texte, voir Lefebvre dans Choix de poèmes, op. cit., p. 13-21. Dans Renverse du souffle, op. cit., p. 187, Lefebvre explique le versant positif de ce titre, l'opposition de Celan « au thème, à la métaphore, au motif, à l'image ». L'image ne disparaîtra pas totalement du dire, mais sera plutôt voilée, infléchie, affranchie des traditions françaises des derniers 
On voit encore une fois que le devenir est ce à quoi on peut se consacrer poétiquement. Cela prend du temps. Il nous attend dans l'épaisseur du dire, à condition que l'on apprenne à construire, à naviguer et à recevoir celle-ci avec soin. S'il y a prière, rites et illumination, ils ont lieu à travers notre approche patiente du poème. Certes, il y a des pages ici qui désorientent, où le sens figuré le dispute en importance au sens propre, lequel semble parfois se volatiliser. Mais, à cet égard, le canon poétique moderne peut ou doit servir de renfort. On peut y trouver des grilles de lecture utiles quant au pouvoir des mots en dehors des structures dites classiques, quelquefois en regardant encore plus loin, vers l'Antiquité. Nous aurons l'occasion d'en parler dans le chapitre 6, à propos des traces de la culture judaïque dans Le Troisième et Éternité à coudre. En outre, le livre-poème Encre plus rouge offre de nombreuses clés à sa propre énigme. Comme c'est souvent le cas chez Tellermann, il y aura des tournants dans le récit, d'autres motifs qui ouvrent sur d'autres sens et qui bouclent la boucle, ainsi que des moments moins impersonnels comme ces vers qui closent une séquence de quatre poèmes dans la deuxième partie d'«État d'urgence», adressés autant aux poètes qu'au lecteur : «Je / porte / je / défais / je / bruis. / Je vous suppose / inquiété relu / neuf » (38).

Quelle est la place des morts dans le récit? Quel équilibre Tellermann établit-elle entre les mots qui font penser à eux et la matière du poème qui interrogera chaque fois plus sur leur présence ? Entre les actions des écrivains du passé et la «caresse » parfois charnelle de sa part, laquelle «tress[e]» et «nou[e] » des liens à l'Autre, met la locutrice en chair et en os avec celui-ci « au bord des étoiles», «dans l'Amen» (93) ? Témoigne-t-on mieux « en rythme fugitif » (144) ? Le texte sert-il mieux de demeure en tant que «parchemi[n] » (93) troué, incomplet, incertain? À quel point l'écriture, où « couleurs deviennent / et sombrent» (211), peut-elle nous conduire au bord des «mondes éteints »

siècles. Pour ce qui est du deuil et de l'étroitesse du dire, voir «les sillages » (ER 22), «à l'endroit d'une étoile / vint la raie» (41), « son d'une vieille terre [...] comme route fragile [...] surgie de l'aveugle / / en boisseaux » (74) et « Dis-lui / les escales les détroits » (94-95). Cf. « L'écluse », La Rose de Personne, op. cit., p. 31, et les poèmes « Il y aura quelque chose, plus tard » et « Baignés de lumière les germes », Enclos du temps / Zeitgehöft, op. cit., p. 87 et p. 97. Quant au renversement chez un autre poète contemporain, voir Régis Lefort, Bernard Vargaftig : esthétique du renversement, Leiden : Brill Rodopi, 2019. Dans $E R$, l'« irruption » fait écho au poème « Wortaufschüttung » / « Remblaiement de mots », Renverse du souffle, op. cit., p. 44-45. 
(116) ? Ce qui nous intéressera dans l'analyse suivante, ce ne sera pas tant d'éventuelles réponses à ces questions, que la façon dont Tellermann les pose au sein de la progression du récit et du dire hautement intersubjectif. Nous nous proposons de montrer que l'adresse au Tu ou au Toi dans les deux prochaines séquences - le sujet lyrique en dialogue avec soi et avec d'autres voix - aide à bâtir une relation entre les morts et les vivants. Par ailleurs, cette polyphonie est ce que l'on peut appeler un « contour mélodique» (UO 92) du recueil. Elle inscrit dans le dire l'échange et la rencontre, suggérant encore une fois que les liens se construisent pas à pas, par le biais du langage. S'y entretissent des voix afin de léguer à d'autres (cf. $E R$ 225) un même élan intersubjectif.

Il s'agit tout d'abord de recueillir les voix d'autres auteurs, lesquelles lui montrent le chemin vers des espaces intérieurs. Rester à l'écoute du dire, c'est aussi se faire guider par ceux qui ont accepté auparavant de «bataill[er] / dur » malgré « le tragique / de deux versants » (ER 77), entrer avec eux dans l'espace sacré des signes. Un aspect important de la progression du récit est la tension entre la vision d'une telle alliance et la difficulté de réaliser ce rêve, de pousser assez loin, de descendre dans ces profondeurs, d'autant que le poète doit dépasser sa solitude. Au-delà des épissures et des séries d'images qui créent des couches de sens, il convient d'étudier de près les nombreux énoncés dialogiques indiquant ce mouvement en avant, ce renouvellement des alliances qui facilitera l'accès aux mondes d'en bas. Pour la plupart, les énoncés sont soit mythiques, soit littéraires et réflexifs. Pour commencer, notons surtout les tensions révélées lorsque Tellermann se demande comment agir. Dans la section «État d'urgence », nous retenons qu'il faut prendre son temps, rester à distance de l'Histoire dans sa noirceur, voir l'« urgence » comme injonction de se dire et non pas de tout dire. Le piquant là-dedans, c'est le relationnel : un chant «superpose» et un « Tu » enjoint de rester sur «les bords» (18). Lorsque nous ne savons pas qui parle à qui, comme dans l'énoncé « - Deviens / je scruterai pour toi / les forêts bleues » (20), Tellermann semble vouloir dire que le côté cérémonial et intersubjectif de l'acte poétique l'emporte sur tout but atteint (cf. 18). On dirait que cette façon ouverte de faire, de penser, de souffler doit sous-tendre nos liens aux morts. La « ferveur» est synonyme de « choses périssables » (43), « la bande d'or» fixée pour incarner des liens fragiles avec un « toi » (44) le contrepoids de tout ce qui est dur et doctrinaire. Là où le Je lyrique semble se parler, c'est pour se rappeler que la tâche d'aborder l'autre rive ne sera jamais facile : «Pour encore tu commences / dans les pôles inverses / d'une seconde terre / ... » (56). ${ }^{49}$

49 Cf. Paul Celan, « Les pôles », [Enclos du temps], in John E. Jackson, Paul Celan : contre-parole et absolu poétique, op. cit., p. 142, où les pôles « sont en nous, / insurmontables / pendant la veille » mais passés « en sommeil ». 
Les deux séquences de la section « Rouge couleur des nerfs » poursuivent ces tensions en parlant plus directement de la mort. Le souffle d'un Nous se trouve affaibli par le grondement de la modernité, alenti par le mystère des plaintes, coupé par la violence physique, mais aussi réuni avec la terre en pleine acceptation des cycles humains et telluriques. Peu de dialogues apparaissent dans la première séquence (61-86), où le regard est assez intériorisé, affrontant l'«à pic » (67) de cette souffrance généralisée. La polyphonie y a lieu à voix basse, provenant par exemple d'un fil baudelairien qui parcourt le livre évoquant - comme dans «Le cygne» - ceux et celles qui regrettent qu'ils soient « captif[s] » et « suppli[ent] » dans une sorte de «stupeur » (63, $68,30) . .^{50}$ Le chant s'assombrit par moments, son dialogisme devenant plutôt allusif, à cause des chocs qui mettent la locutrice dans un état méditatif, état où elle s'identifie à un «nous couchés entre les soldats » (69) et au fait de devoir « quitt $[\mathrm{er}][\ldots]$ notre nom » (70). Mais, en même temps, elle se réjouit d'une « fable inversée » grâce à laquelle les morts participent de manière surgissante et généreuse au dire (73), ou bien fait appel au thème hölderlinien des « fêtes [qui] ont leurs saisons » et « font descendre le rayon » (78) - celui, chez Hölderlin, d'une langue renaissante, nimbée de l'or des choses simples et de l'amitié. ${ }^{51}$ En effet, une «sainteté » (82) ambivalente semble être la résultante de ce dire mouvementé : soit parce que le «rouge couleur des nerfs » représente le sang symbolique coulant dans les veines du poète lorsqu'il a affaire au tragique, soit parce que le chant affirme que le tragique peut être dépassé par le chant. L'acharnement, suggéré par le vers « Ô meute / appendue au rêve » (86), résume en tant que formule clé la première séquence, et ceci dans plusieurs sens : les conditions qui font que les humains veulent tuer, la violence et la souffrance qu'il nous faut combattre, l'aspect conflictuel du désir d'appartenir à un Nous et les diverses voix qui se joignent toujours au dire, celles des morts et des vivants, des poètes d'aujourd'hui et des jours révolus.

Ladresse au Tu ou au Toi se fait particulièrement insistante dans la deuxième séquence $(87-103)$. Le féminin, très présent dans l'avant-dernier vers de la première séquence, «Or jadis / elle aima » (86), joue un rôle important : ces

50 « [T] retirent Suwavna / aux Afriques et cocotiers brumeux» (30); « le lent repos / aux Afriques superbes» (198); « les captifs rauques» (150); « chaque fois / plus rauque la voix » (84); «Sommes captifs / quelle que soit la couleur » (170). Cf. Charles Baudelaire, Les Fleurs du mal, éd. Yves Florenne, Paris : LDP, 1972, p. 211-13, par exemple vis-à-vis des « cocotiers absents de la superbe Afrique » (213). Hopkins, « No worst, there is none » / « Non, de pire, rien », Grandeur de Dieu, trad. et éd. Jean Mambrino, Orbey : Arfuyen, 2005, p. 78-79. 
pages, adressées souvent à Celan, expriment le souhait de refaire avec lui un destin meilleur, moins cruel et plus prometteur. Tellermann s'appuie sur le rêve pour aider son confrère, renoue avec le chant comme recherche d'un méridien, de points de contact avec l'Autre en dépit des distances qui le séparent du moi. Alors que le cadre précédent était souvent celui d'un « décembre chaud » $(74,76,83)$, cette séquence est relativement ensoleillée. Cet ensoleillement correspond au désir de définir la femme autrement (89), en tant que plénitude par exemple « éployée dans l'Amen » (98), en dehors des traditions sociales ou religieuses qui peuvent l'obliger à suivre le vouloir de l'homme. ${ }^{52}$

Considérons la communion qui s'effectue dans la parole vis-à-vis des exhortations à soi et du souci de seconder Celan. L'aveuglement (26, 90, 96) est un mot clé, emblématique de la parole poétique dans son ouverture au silence et au non-dit comme aux seuils et aux méridiens : « Nous échangions des mots / d'aveugle / à jamais / confondus / à jamais / divisibles » (96). Or, cet aveuglement mène au dialogue et à l'introspection, à l'échange et aux rencontres, au songe et à la découverte. Est « aveugle » le poète qui se sait incapable de se lier véritablement aux morts, voué à les regarder d'une autre rive. L'adresse au Tu cible Celan à propos de ce dilemme, mais, pour ainsi dire, rebondit sur Tellermann, comprend celle-ci dans le désir de toujours faire mieux, de ne pas abandonner, de « cultive[r] la racine / suppliée dans le voile » (97), de reconnaittre ce qui ne pourra se dire et d'en parler tout de même. Ce non-dit, cette dureté de l'indicible, se nomme ici «acier sous les paupières » (97), reprise d'un vers celanien concernant le devoir de continuer à entrevoir, à creuser : « Il y aura un cil, / tourné vers le dedans de la roche, / durci à l'acier du non-pleuré, / le plus fin de tous les fuseaux. $»^{53}$

En effet, il s'agit dans cette séquence de « creus[er] » (95) et de « cultive[r] » (97), de continuer à regarder au-dedans de soi - à travers le songe, le mythe et

52 «[F]emme définie selon la source / le Dieu qu'on suppose / une ligne vide au travers » (ER 89). Cf. Aaron Prevots, «Entretien avec Esther Tellermann », op. cit., p. 106 : « Comment avoir une parole autre que celle qui se moule sur celle de l'homme ? Ce sont ces interrogations qui m'ont sans doute hantée, comme me hante, après l'Ophélie de Shakespeare celle de Nerval. Une femme cherche-t-elle au fond à être rejetée de la parole ? À flotter couronnée sur les courants?»

53 Paul Celan, «Confiance», [Grille de parole], Choix de poèmes, op. cit., p. 131. Le titre allemand de ce poème, «Zuversicht», connote l'optimisme et l'œil fin de celui qui voit en avant, à travers, au-dedans, au-delà. Signalons d'ailleurs qu'une telle confiance peut avoir lieu puisqu'elle est axée sur l'intersubjectif. C'est du contexte celanien que vient l'idée de l'adresse au Tu ou au Toi comme grille analytique, ainsi que celle d'une advenue à soi qui s'effectue à travers cette adresse à l'Autre. Voir John E. Jackson, Paul Celan : contre-parole et absolu poétique, op. cit., p. 41 et p. 50 du chapitre 2, « Le principe dialogique » (41-73). 
les traditions littéraires - et d'aider autrui à mieux voir aussi. Les nombreux impératifs nous rappellent ceux des deux premiers textes en regard, ainsi que le caractère charnel de l'acte poétique et les effets sonores créés par des répétitions si volontaires : «Assouvis la caresse » (93) ; « Dis-lui » (94, 95); « Écris » $(89,93,102)$; « Entends » $(97)$; « Reste » $(91,92) ;$ « Va plus loin » $(100)$; «Vois » (101). Juxtaposé à ces impératifs, le mot composé «bouche-sœur» (9o) en résume les enjeux. Tellermann s'adresse autant à elle-même quà Celan. Elle intègre celui-ci dans une famille poétique. Elle reprend certains de ses motifs pour insister sur l'espoir et une recherche à deux, une parole commune. En particulier, elle intercale dans le récit l'idée celanienne que ceux et celles ayant conscience de la catastrophe se doivent de la représenter dans leurs écrits, à la fois pour partager des émotions et mener plus loin la discussion. Là où Celan aurait généralement écrit à une femme, Tellermann inverse le cadre en faisant de Celan momentanément son interlocutrice, «sœur » ou proche métaphorique : « je t'appellerai Ruth / l'étrangère / nous labourerons / les seuils » $(92 ; \mathrm{cf}$. 100). ${ }^{54}$ Ces pages nous rappellent qu'il faut sauvegarder le mot multiple - énigmatique, ambivalent, polysémique - échangé avec l'Autre, quand bien même « la peur » (102) aurait pénétré dans le dire..$^{55}$ Elles nous montrent que l'adresse à l'Autre se poursuit mot par mot, étape par étape, au fur et à mesure qu'une relation - parfois ludique - se développe. À cet égard, après tous les encouragements, questions et réponses de cette deuxième séquence, la fin mène vers le contact avec ceux et celles qui ont disparu se faisant « travers[ée]» (103) de continents qui ne peut prendre fin.

$54 \mathrm{Au}$ sujet de la métaphore du poète comme «bouche» qui échange des «mot[s] d'aveugle » (ER 9o), voir par exemple Paul Celan, «Grille de parole», [Grille de parole], Choix de poèmes, op. cit., p. 139 : « deux / pleines bouches de silence ». Cf. « Les cruches », [Pavot et Mémoire], ibid., p. 69: «elles portent le vide à la bouche comme le plein »; «Sibérien», [La Rose de Personne], ibid., p. 195: « galet blanc dans la bouche». En ce qui concerne le mot composé tellermannien «bouche-sœur» (9o), voir «Je suis seul», [Pavot et Mémoire], Choix de poèmes p. 67, puis «Chymique», [La Rose de Personne], p. 183, à propos d'une « silhouette-sœur ». Quant aux échanges épistolaires de Celan avec Nelly Sachs en tant que « sœur » métaphorique ou « bouche » avec qui parler, voir en particulier « Zürich, Zum Storchen » et « Lécluse », La Rose de Personne, op. cit., p. 17 et p. 31, ainsi que John Felstiner, Paul Celan : Poet, Survivor, Jew, op. cit., p. 161-62. Les vers sur Ruth sont un clin d'œil au poème «En Égypte », [Pavot et Mémoire], Choix de poèmes p. 61 : « Tu chercheras dans l'œil de l'étrangère celles que tu sais dans l'eau. / Tu les appelleras : Ruth ! Noémie ! Myriam ! pour qu'elles sortent de l'eau. »

Cf. Paul Celan, «L'écluse », La Rose de Personne, op. cit., p. 31, où « sœur » est un « mot multiple». 
La troisième partie du recueil, «Encre plus rouge » (107-228), met en avant les «mondes éteints » (116) et introduit ainsi d'autres tonalités. Elle continue à bâtir un Nous, à travers un passé de plus en plus mythique. Nous voyons de nouveau qu'il s'agit autant d'un récit sur l'inconscient et ses liens à la littérature - «nos fables » (109), l'encre comme chemin vers ceux et celles de l'audelà - que sur les morts eux-mêmes. L'idée de «contour[s] mélodique[s] » ( UO 92) s'impose, outil critique utile quant aux pas faits vers l'Antiquité et ses figures, et au dépliement spatiotemporel qui continue à s'inscrire dans le dire. Ce dépliement et le devenir humain qui s'y intrique sont soulignés par les leitmotivs du « signe mat et clair » $(E R$ 188, 191, 195, 204, 219) et du poète gardien du signe (16o ; cf. 35). En quelque sorte, l'adresse au Tu ou au Toi s'enrichit par l'ambivalence de ce Tu, c'est-à-dire le manque de références précises aux interlocuteurs-silhouettes et leur entrelacement dans une prière diffuse qui couvre un champ si vaste. On voit que le signe est « mat et clair » parce qu'il devient nécessité d'avancer ensemble humblement en « cogn[ant] nos genoux » (195), d'acquiescer au signe comme « source » $(195,219)$, d'accepter que la présence de l'Autre ne se sépare pas de la recherche de cet Autre, de la question « [O]ù es-tu $[\ldots]$ ? » (197).

Il convient d'examiner quelques derniers exemples de cette adresse au Tu ou au Toi, ainsi qu'au Vous. Globalement, on peut parler d'« offrandes » (66) qui continuent à « s'égrener » (42) au moyen d'images brèves et cristallines dans un monde polyreligieux, sans foi précise. ${ }^{56}$ Lorsque Tellermann dit, « offre la gorge et l'encre / la fable qui pourvoit / / et je te façonne en songe » (222), le Tu peut finalement correspondre à un éventail d'auteurs, de Perse et Celan à Hölderlin et Du Bouchet, à Hopkins et Nerval, ou bien aux trépassés de toutes sortes qui nous habitent au travers du signe. ${ }^{57}$ Lorsqu'il

56 Cf. Jean Richer, Gérard de Nerval, Paris : Seghers, 1972, p. 63, au sujet de «l'absence d'une foi précise » chez Nerval.

À ce stade du récit, les allusions s'imbriquent, et donc les éventuelles couches interprétatives aussi. L'image du « soleil noir» $(E R$ 141, 200), dont nous avons traité à propos de Perse (Vents, III, 5, p. 228 : «Et révérence au Soleil noir d'en bas !»), peut être liée au poème «El Desdichado» de Nerval (Richer, op. cit., p. 101). Elle peut également faire penser aux références aux portes et à Jérusalem chez Nerval ou Celan, et ainsi de suite (Nerval, Aurélia, II, « Mémorables » : « Quand sa houssine légère toucha la porte de nacre de la Jérusalem nouvelle, nous fûmes tous les trois inondés de lumière », Richer, op. cit., p. 154). L'allusion à la page 200 - « soleil noir s'est levé / aux portes de Jérusalem » - est au poème « Cette nuit est irrémédiable » d'Ossip Mandelstam ; voir Paul Celan, « La poésie d'Ossip Mandelstam », trad. Bertrand Badiou, Po\&sie 52 (1990), p. 9-20 (15-16), <po-et-sie. fr>. Autre clin d'œil à Hölderlin, en dehors du «rayon » (78) dont nous avons traité : la «neige désaccordée » $(E R$ 167). 
traque quelqu'un «dans l'entaille», le poème traite d'un Tu qui se transforme en Vous et qui englobe ainsi tous les disparus, peut-être ceux et celles qui prient les paumes ouvertes pour que jour et nuit s'équilibrent grâce aux « équinoxes » (154): «Qui es-tu / qui n'es-tu pas / toi traqué dans l'entaille / / vous / les cheveux au travers » (155). ${ }^{58}$ Les rites dépeints - « Écoute encore » (154) - font écho aux premiers mots du livre, mais les brûlures des premières pages $(21,35,43)$ ont une portée encore plus vaste, celle de rites antiques pour reconnaître une Salomé, une Iphigénie, un Ange (201, 202, 203, 212, 213, 218), ainsi que tout ce qui se métamorphose dans la terre pour, de même, colorer notre vie, tels le violet, l'ambre et les micas (213). Quelques vers en particulier sont révélateurs quant à la boucle du récit bouclée pour l'instant, aux liens intersubjectifs entre le moi et les morts laissés derrière soi pour s'asseoir (42) dans une autre sorte de cercle, celui de collectivités d'aujourd'hui : «Je vous bercerai en songe / à moi / et pour toujours / l'Un seul / et je retourne dans le gel / m'assois parmi les vivants » (203). Autre rite primordial pour clore le livre en l'ouvrant sereinement: se dévouer à un Toi féminin, un Toi qui se métamorphose en Elle, à qui offrir « la rose / / de Sijilmassa » $(217$; cf. 215, 226), façon visuelle d'être dans le don tout comme celle également sonore concernant «la sonate » à disperser « au fond rouge / dans toute chose / qui tombe » $(227$; cf. 201, 218).

Ettre «davantage » $(161,187,211)$ : voilà qui est au cœur de la polyphonie d'Encre plus rouge. Le rouge, c'est ce fil de la pensée tendu vers soi et l'Autre, pour voir comment le désir va se nuancer, quelles relations vont en ressortir. On ne retrouve pas forcément un seul sens déterminé, ni la pensée «parvenue » (211). Tellermann va loin dans le temps et l'espace, mais garde en même temps ses distances vis-à-vis des rapports qu'elle voudrait révéler par le signe. Aux morts de reconnaître le respect qui sous-tend ces gestes. Au lecteur d'accepter ce regard qui vise l'Autre à travers une grille de parole, un Toi «sur le point d'être dit » (210). Les « rouleaux » (43) du récit se déploient en séquences, mais aucun thème n'est privilégié : il faut accueillir les couleurs allégoriques du dire,

$5^{8} \quad$ Notons aussi l'allusion à l'Antiquité : « Les deux mains dressées, la paume en avant (passis manibus selon les termes de César, dans la Guerre des Gaules) sont un geste de supplication ; les Gauloises en usent à plusieurs reprises au cours de la Guerre des Gaules [...] avec ou sans dénudement du sein » (Jean Chevalier et Alain Gheerbrant, éd., Dictionnaire des symboles, op. cit., p. 6o1). Il est également possible de voir dans cette image de la main ouverte une allusion à André du Bouchet pensant à Hölderlin, à Celan et au fait d'entrer dans la langue comme sacrement par lequel on saisit celle-ci la main ouverte ; voir Tübingen, le 22 mai 1986, in André du Bouchet, ...désaccordée comme par de la neige et Tübingen, le 22 mai 1986, Paris : Mercure de France, 1989, p. 51-93 (55-56). 
traverser les scènes de divers conflits, songer, veiller, bercer, encore et encore. Les variations se font écho et s'entrecroisent. La musique de ces variations est sens. ${ }^{59}$ Les figures sonores n'en finissent pas de nous éclaircir, jusqu'à l'os et à la chair de l'« être corporel travaillé par la mort ». ${ }^{60}$

Activer des chaînes associatives devient ainsi une expédition vers la vérité de l'Autre et du langage, une découverte à la fois des périphéries sémantiques de mots à la tonalité profonde et des relations humaines que ceux-ci établissent. ${ }^{61}$ Pour Tellermann, c'est une quête ayant lieu dans la mémoire et l'inconscient, mais ancrée dans la tradition littéraire. L'étoffe allusive et polysémique du dire a une allure vibratoire qui exige que le lecteur prête son concours au déchiffrement. Encre plus rouge nous montre que chanter les morts, c'est rester disponible non seulement à l'« inattendu » du signe ${ }^{62}$ et aux affres de l'Histoire, mais aussi aux « énergies latentes dans les œuvres du passé ».63 S’inscrivant dans un ordre du devenir à la fois littéraire, mythique, linguistique et ontologique, ce

59 Cf. Esther Tellermann, « Autour de la lecture de Michèle Montrelay et Jacques Lacan de Lol V. Stein de Marguerite Duras », Journal français de psychiatrie 43 (2016/1), p. 138-40 (138). Voir aussi Isabelle Garron, «Esther Tellermann : Encre plus rouge », op. cit., p. 74-75 (74) : « [L]'univers "tellermannien" interroge le poème en son principe de réalité le plus flagrant : le vers. Un vers antique presque, dont les interrogations, les coupes, la présence d'Iphigénie, d'autres villes ou femmes mythiques ravivent une lyrique racinienne; sa rigueur prosodique telle la chair des tragédies. »

6o Jean-Marie Perret, « Esther Tellermann, Encre plus rouge », op. cit., p. 114.

61 Nous empruntons à Jean-Pierre Lefebvre les notions de « reconnaître [...] les périphéries sémantiques de certains mots » et de faire ce que Kafka nomme « une expédition vers le vrai » (Paul Celan, Choix de poèmes, op. cit., p. 18 et p. 19). Quelques références de la part de Tellermann vont dans ce dernier sens, lorsqu'elle parle du « Nord » (ER 49) ou de « choucas » $(17,42)$. En tchèque, Kafka veut dire choucas, petite corneille noire qui « hante les pierriers d'altitude » (Paul Celan, Partie de neige, trad. et éd. Jean-Pierre Lefebvre, Paris : Seuil, 2013, p. 182n8). De telles paronomases ont lieu aussi chez Celan. L'enjeu est pourtant grave : celui d'habiter haut dans le ciel par le poème les lieux où la cendre devient neige, lieux qu'habitent par moments des écrivains du nord comme Ossip Mandelstam ou Marina Tsvétaeva. Voir ER 141, " aria sur / la neige incandescente / il gèle dur », et Choix de poèmes, op.cit., p. 229 et p. 322 . Selon Lefebvre, le nord peut être le « royaume des morts » (Choix de poèmes, op. cit., p. 36on2).

62 À propos du «lien de pure vacance aux signifiants, cette disponibilité à la trouvaille, à l'inattendu, où le signifiant montre son autre face », voir Esther Tellermann, « Autour de la lecture de Michèle Montrelay et Jacques Lacan de Lol V. Stein de Marguerite Duras », op. cit., p. 140 .

63 Collectif, Passeurs de mémoire : de Théocrite à Alfred Jarry, la poésie de toujours lue par 43 poètes d'aujourd'hui, préface de Jean-Baptiste Para, Paris: Poésie/Gallimard, 2005, p. 12. Une remarque de Tellermann va dans le même sens : «que le souffle soit mémoire » (NN 202). 
texte nous enseigne sur nous-mêmes en tant qu'êtres créés par la portée de nos cultures et identités en même temps que sur celles de l'Autre. Aussi voit-on l'ontos étroitement lié à la prière qui soulage, aux rites à la lisière de l'union, et à l'éthique d'un Nous qui se réalisera également «à l'envers» du «grand Livre » (ER 228), dans la vraie vie. 